\title{
Guía de profilaxia y tratamiento antitrombótico para pacientes con COVID-19. Consenso del Grupo Cooperativo Latinoamericano en Hemostasia y Trombosis (CLAHT)
}

\author{
Guidelines for prophylaxis and anti-thrombotic treatment for patients with COVID-19. \\ Consensus of the Latin American Cooperative Group on Hemostasis and Thrombosis \\ (CLAHT)
}

\begin{abstract}
Cecilia Guillermo-Esposito', Patricia Casais², Gabriela Cesarman-Maus³, Fernanda Andrade-Orsi', Rosario Martínez-Rovira ${ }^{5}$, Joao C. de-Campos-Guerra ${ }^{6}$, Suely M. Rezende ${ }^{7}$ y Raúl Izaguirre-Ávila ${ }^{8 *}$

${ }^{1}$ Cátedra de Hematología, Facultad de Medicina, Hospital de Clínicas, Universidad de la República, Montevideo, Uruguay; ${ }^{2}$ Departamento de Epidemiología Clínica y Evidencia, Instituto de Investigaciones en Salud Pública, Universidad de Buenos Aires, Argentina; ${ }^{3}$ Departamento de Hematología, Instituto Nacional de Cancerología, Ciudad de México, México; ${ }^{4}$ Departamento de Patología Clínica, Escuela de Ciencias Médicas, Universidad de Campinas, Campinas-Sao Paulo, Brasil; ${ }^{5}$ Unidad de Hemostasis y Trombosis, Hospital de Clínicas, Universidad de la República, Montevideo, Uruguay; ${ }^{6}$ Hospital Israelita Albert Einstein, Centro de Hematología de Sao Paulo, Sao Paulo, Brasil; ${ }^{7}$ Facultad de Medicina, Universidad Federal de Minas Gerais, Belo Horizonte, Minas Gerais, Brasil; ${ }^{8}$ Departamento de Hematología, Instituto Nacional de Cardiología Ignacio Chávez, Ciudad de México, México

Panel de colaboradores (por país)t: Argentina: Raúl Altman, José Ceresetto, Cecilia Colorio, Cristina Duboscq, Claudio Martín Rosa, Soledad Molnar, Diana García; Bolivia: Mario Luis Tejerina, Nelson Nina García, Juan Choque Pacheco; Brasil: J. María Chiara Chindamo; Colombia: Gloria Ramos Ramos, Guillermo León Basantes, Aura Milena Reyna, Miriam Cantero Guevara; México: Mateo Porres Aguilar, Luis Meillón, Flavio Adrián Grimaldo; Nicaragua: Ludwing Alexander Bacon Fonseca; Panamá: Al Ávila Milord, Ricardo Aguilar, Rafael Aparicio; Paraguay: Paula de Guggiari; Perú: Gloria Chumpitaz, Pedro Pablo García; Uruguay: Blanca Steffano, Sandra Damiano; Venezuela: Juan Serrano, Marisol Zavahra.

"Todos los autores han contribuido equitativamente en la elaboración de este documento.
\end{abstract}

\section{Resumen}

La coagulopatía y la trombosis asociadas a la enfermedad por coronavirus 2019 (COVID-19) representan un problema importante en el manejo de esta enfermedad. Los estudios clínicos de los últimos meses han demostrado que los pacientes con COVID-19 presentan un estado de hipercoagulabilidad particular, en el que se observa un aumento notable del dímero $D$ concomitante con niveles elevados de fibrinógeno. El estado de hipercoagulabilidad conduce a un mayor riesgo de trombosis, que parece ser mayor entre aquellos pacientes con síntomas críticos de COVID-19. El mejor enfoque terapéutico para prevenir los eventos trombóticos en esta nueva enfermedad aún no se ha determinado y han surgido varias preguntas con respecto a la tromboprofilaxia, como el momento adecuado para iniciar la anticoagulación, el tipo de anticoagulante y el régimen de dosis. Para abordar estas preocupaciones, varias sociedades médicas han publicado artículos de posición para brindar la opinión de expertos en trombosis sobre el manejo de la coagulopatía y trombosis asociadas a COVID-19. EI Grupo Cooperativo Latinoamericano de Hemostasia y Trombosis (Grupo CLAHT) ha convocado a un panel de expertos en trombosis y hemostasia para discutir los datos disponibles sobre este tema. El objetivo de esta revisión es resumir la evidencia actual con respecto al deterioro hemostático y el riesgo trombótico en el COVID-19 y proporcionar una opinión cui-

\footnotetext{
Correspondencia:

*Raúl Izaguirre-Ávila

E-mail: rizagui@yahoo.com

Fecha de recepción: 16-06-2020

Fecha de aceptación: 17-08-2020

DOI: $10.24875 /$ ACM.20000291

1405-9940 / @ 2020 Instituto Nacional de Cardiología Ignacio Chávez. Publicado por Permanyer. Este es un artículo open access bajo la licencia
} 
dadosamente revisada de los expertos latinoamericanos sobre la tromboprofilaxis y el manejo de eventos trombóticos y coagulopatía en pacientes con sospecha de COVID-19.

Palabras clave: COVID-19. Tromboprofilaxia. Trombosis. Tromboembolia. SARS-CoV-2. Neumonía.

\begin{abstract}
Coagulopathy and thrombosis associated with coronavirus disease 2019 (COVID-19) represent a major issue in the management of this disease. In the past months, clinical studies have demonstrated that COVID-19 patients present with a particular hypercoagulable state, in which a markedly increased D-dimer concomitant with increased levels of fibrinogen are observed. This hypercoagulable state leads to an increased risk of thrombosis, which seems to be higher among those patients with critical symptoms of COVID-19. The best therapeutic approach to prevent thrombotic events in COVID-19 has not been determined yet and several questions regarding thromboprophylaxis therapy, such as the time to initiate anticoagulation, type of anticoagulant and dose regimen, have emerged among physicians. To address these concerns, several medical societies have published position papers to provide the opinion of thrombosis experts on the management of coagulopathy and thrombosis associated with COVID-19. In line with this, the Latin America Cooperative Group of Hemostasis and Thrombosis (Grupo CLAHT) has constituted a panel of experts in thrombosis and hemostasis to discuss the available data on this topic. The aim of this review is to summarize the current evidence regarding hemostatic impairment and thrombotic risk in COVID-19 and to provide a carefully revised opinion of Latin American experts on the thromboprophylaxis and management of thrombotic events and coagulopathy in patients with suspected COVID-19.
\end{abstract}

Key words: COVID-19. Thromboprophylaxis. Thrombosis. Thromboembolism. SARS-CoV-2. Pneumonia.

\section{Introducción}

La enfermedad por coronavirus del 2019 (COVID-19) es una infección viral aguda secundaria al coronavirus tipo 2 del síndrome respiratorio agudo grave (SARSCoV-2, Severe Acute Respiratory Syndrome Coronavirus 2). Alrededor del $80 \%$ de los pacientes con COVID-19 tiene síntomas leves a moderados, 14\% desarrolla enfermedad grave (disnea, taquipnea, hipoxia) y cerca del $6 \%$ progresa a un estado crítico (insuficiencia respiratoria, choque séptico o disfunción multiorgánica) ${ }^{1}$. La respuesta inflamatoria sistémica no controlada, resultado de la liberación de grandes cantidades de citocinas proinflamatorias, es el mecanismo subyacente esencial del síndrome respiratorio agudo grave y la insuficiencia orgánica múltiple, principales causas de muerte de la COVID-192.

En relación concomitante con el estado inflamatorio, las personas con infección por SARS-COV-2 desarroIlan un estado hipercoagulable. Varios estudios han descrito alteraciones de la hemostasia en la COVID-19. El estado de hipercoagulabilidad se caracteriza por elevación de los valores de fibrinógeno, dímero D, trombocitosis y acortamiento del tiempo de tromboplastina parcial activado (TTPa) provocado por cifras elevadas del factor VIII (FVIII) y el factor de von Willebrand $(\mathrm{fvW})^{2-6}$. En etapas más avanzadas puede
Tabla 1. Escala de CID de la International Society on Thrombosis and Haemostasis (ISTH)

\begin{tabular}{|l|c|c|}
\hline Variable & Valor & Puntuación \\
\hline \begin{tabular}{l|c} 
Recuento plaquetario \\
$\left(10^{3} / \mu \mathrm{L}\right)$
\end{tabular} & $\begin{array}{c}50 \text { a } 100 \\
<50,000\end{array}$ & 2 \\
\hline $\begin{array}{l}\text { Prolongación del tiempo de } \\
\text { protrombina (segundos en } \\
\text { relación con el valor de }\end{array}$ & $3-6$ & 1 \\
referencia) & $>6$ & 2 \\
\hline Fibrinógeno $(\mathrm{mg} / \mathrm{dL})$ & & 1 \\
\hline Dímero D (ng/mL) & $<100 \mathrm{mg} / \mathrm{dL}$ & 1 \\
\hline
\end{tabular}

*Una puntuación $\geq 5$ indica CID.

registrarse ligera prolongación del TP y el TTPa, así como discreta trombocitopenia (100-150/L). Los signos de coagulación intravascular diseminada (CID) y el aumento del dímero $\mathrm{D}$ se relacionaron con un mayor riesgo de muerte en una cohorte china en Wuhan?

El estado de hipercoagulabilidad secundario al SARS-CoV-2 se ha vinculado sobre todo con un riesgo elevado de enfermedad tromboembólica venosa (ETEV), pero también con trombosis arteriales. Por otra parte, como se ha demostrado en series de necropsias, la infección induce vasculopatía y trombosis de 
pequeños vasos, de manera predominante en el parénquima pulmonar (algunos especialistas lo denominan en la actualidad coagulopatía intravascular pulmonar), aunque en etapas avanzadas puede afectar la microvasculatura en otros sitios, como piel o riñones ${ }^{8-11}$. Se ha notificado una elevada incidencia de trombosis a pesar de la administración de dosis profilácticas de heparinas, en particular en pacientes en la unidad de cuidados intensivos $(\mathrm{UCl})^{12-14}$. Algunos autores han observado una incidencia acumulada de trombosis durante la hospitalización superior al $25 \% \%^{12,15-17}$, que supera valores de $40 \%$ en pacientes en estado crítico ${ }^{13,18}$. Además, hasta la mitad de las trombosis puede detectarse en las primeras $24 \mathrm{~h}$ de la hospitalización ${ }^{17}$. Se ha sugerido que la heparina podría incrementar la supervivencia de los pacientes con COVID-19 crítico'. En conclusión, los estudios señalan un mayor riesgo trombótico, similar o incluso mayor que el observado en individuos críticos sin COVID-19, a pesar de la profilaxia antitrombótica.

La evidencia clínica sugiere hasta ahora que la prevención de la trombosis sería una pieza vital del tratamiento integral de los pacientes con COVID-19. Sin embargo, hasta la fecha no hay estudios controlados/ aleatorizados ni evidencia científica sólida acerca del mejor esquema terapéutico antitrombótico en estos pacientes. En consecuencia, el grupo Cooperativo Latinoamericano de Hemostasia y Trombosis (CLAHT) reunió un panel de expertos representantes de diferentes sociedades científicas e instituciones de Latinoamérica con la finalidad de revisar la evidencia actual y sugerir medidas de tromboprofilaxia y tratamiento de los episodios trombóticos en pacientes con sospecha de COVID-19, adaptadas a las características de la región. Estas recomendaciones se aplican a la fecha de preparación de este documento y deben revisarse de manera periódica, ya que ésta es una enfermedad nueva y se hallan en curso numerosos estudios aleatorizados.

\section{Metodología}

Se designó a un comité de redacción constituido por siete miembros de cuatro países del grupo CLAHT, que se encargó de la búsqueda y revisión de las publicaciones médicas (fecha de la última actualización bibliográfica: 25 de mayo de 2020). Se invitó a las sociedades de hemostasia y trombosis y de hematología de Latinoamérica y otras instituciones médicas a aportar sus propias guías terapéuticas. Se convocó a una reunión virtual con expertos y representantes de sociedades científicas para la valoración de las
Tabla 2. Escala de coagulopatía inducida por sepsis (CIS) de la International Society on Thrombosis and Haemostasis

\begin{tabular}{|l|l|c|c|c|}
\hline & Parámetro & $\mathbf{0}$ puntos & $\mathbf{1}$ punto & $\mathbf{2}$ puntos \\
\hline $\begin{array}{l}\text { Tiempo de } \\
\text { Protrombina }\end{array}$ & TP-INR & $\leq 1.2$ & $>1.2$ & $>1.4$ \\
\hline Coagulación & $\begin{array}{l}\text { Cuenta de } \\
\text { plaquetas } \\
\left(10^{3} / \mu \mathrm{L}\right)\end{array}$ & $\geq 150$ & $<150$ & $<100$ \\
\hline FOS Total & Suma de 4 & 0 & 1 & $\geq 2$ \\
\hline
\end{tabular}

$\geq 4$ puntos: diagnóstico de coagulopatía inducida por sepsis. FOS: falla orgánica secuencial.

El total de FOS es la suma de la FOS respiratoria, cardiovascular, hepática y renal. TP-INR: tiempo de protrombina expresado en INR.

Tabla 3. Contraindicación para tromboprofilaxia (modificado a partir de Nice Clinical Guidelines 92Venous Thromboembolism 2019 y de la guía ASH para COVID-19)

Uno o más de los siguientes factores de riesgo (a menos que el
riesgo de ETEV sea mayor que el riesgo de hemorragia)
- Hemorragia
- Anomalía hemorrágica adquirida
- Administración concomitante de anticoagulantes
- Anestesia por punción lumbar, epidural o espinal en las
$\quad$ siguientes $12 \mathrm{~h}$
- Anestesia por punción lumbar, epidural, espinal en las $4 \mathrm{~h}$
previas
- Accidente vascular hemorrágico cerebral agudo
- Trombocitopenia $<25 \times 10^{3} / \mu \mathrm{L}$
- Hipertensión sistólica no controlada: $\geq 230 / 120 \mathrm{mmHg}$
- Enfermedad hemorrágica hereditaria no tratada

publicaciones científicas hasta el momento actual y las guías locales e internacionales.

En esta reunión (27 de abril de 2020), en la que participaron 80 asistentes (datos de contacto: 44), se analizaron los temas sobre los que no se dispone de datos y se realizó una primera aproximación al consenso. Además, se compararon las siguientes guías latinoamericanas disponibles al momento de la reunión: Sociedad Argentina de Hematología (versión en línea), Associação Brasileira de Hematología e Hemoterapia y Sociedade Brasileira de Trombose e Hemostasia (aportada por los autores), Sociedad Boliviana de Hemostasia y Trombosis (aportada por los autores), Sociedad Paraguaya de Hematología (versión en línea compartida en Twitter), Sociedad Mexicana de Trombosis y Hemostasia (aportada por los autores) y Pautas de la Unidad de Hemostasis y Trombosis del Hospital de Clínicas de Montevideo, Uruguay (aportada por los autores). 


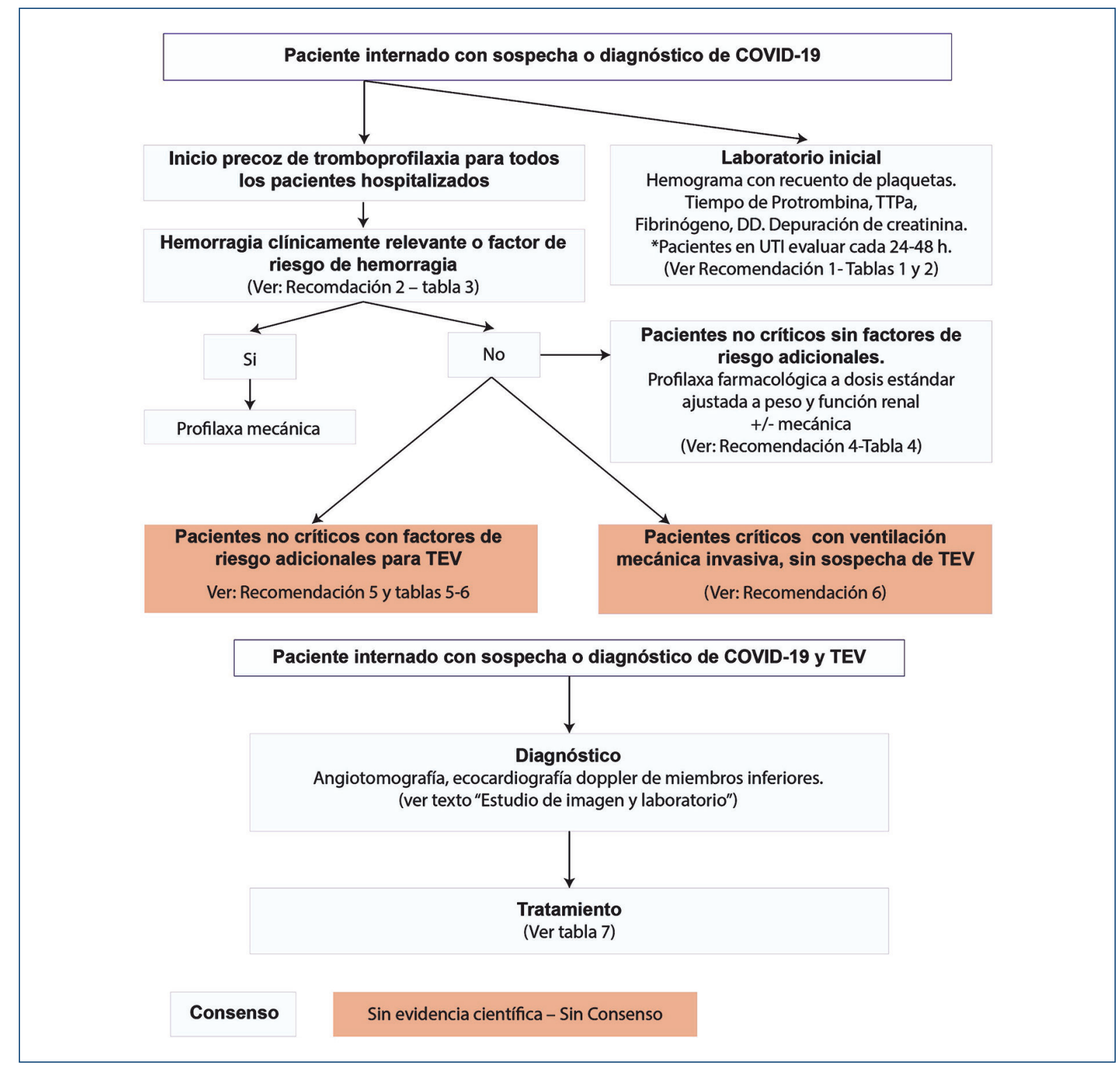

Figura 1. Algoritmo de manejo antitrombótico del paciente internado con sospecha o diagnóstico de COVID-19. TTPa: Tiempo de Tromboplastina Parcial activado; DD: Dímero D; TEV: tromboembolia venosa.

Las conclusiones de la reunión virtual se recogieron en un manuscrito enviado (9 de mayo de 2020) para su revisión a todos los representantes nacionales del CLAHT y a algunos de los expertos participantes de la reunión (quienes enviaron datos de contacto y manifestaron interés en revisar el manuscrito). Se invitó a los que recibieron el documento a compartirlo con otros expertos que desearan ofrecer sus comentarios. Este correo electrónico se envió a 36 personas y se obtuvieron 15 respuestas.

El comité de redacción, luego de valorar las respuestas y sugerencias, redactó un nuevo escrito que incluyó también las guías de Colombia (aportada por sus autores), Panamá (aportada por sus autores) y la versión completa de las guías paraguayas. Este documento se remitió (18 de mayo de 2020) a los miembros del grupo CLAHT $(n=386)$ para su revisión y de nueva cuenta se invitó a compartirlo con expertos que quisieran emitir comentarios. Se obtuvieron 20 respuestas. Además, se realizó una encuesta en línea entre los participantes acerca de algunos temas sobre los que no existe consenso y a la que respondieron 90 personas.

Las recomendaciones o sugerencias de este documento se basan en las publicaciones internacionales 
Tabla 4. Dosis regular de profilaxia con HNF, HBPM y fondaparinux con ajustes de acuerdo con peso y función renal

\begin{tabular}{|c|c|c|c|}
\hline & Dosis regular & Optimización por peso & Insuficiencia renal \\
\hline Enoxaparina & $40 \mathrm{mg} \mathrm{SC}$ cada $24 \mathrm{~h}$ & $\begin{array}{l}80-99 \mathrm{~kg}: 40-60 \mathrm{mg} \mathrm{SC} \text { cada } 24 \mathrm{~h} \\
\geq 100 \mathrm{~kg}: 80 \mathrm{mg} \mathrm{SC} \text { cada } 24 \mathrm{~h}\end{array}$ & $\begin{array}{l}\text { CICr } 15-29 \mathrm{~mL} / \mathrm{min}: \\
\text { Reducir dosis al } 50 \% \\
\mathrm{CICr}<15 \mathrm{~mL} / \mathrm{min}: \text { contraindicada; usar HNF }\end{array}$ \\
\hline Nadroparina & $3,800 \mathrm{UI} \mathrm{SC}$ cada $24 \mathrm{~h}$ & $>70$ kg: 5,700 UI SC cada $24 \mathrm{~h}$ & $\begin{array}{l}\text { CICr } 30-50 \mathrm{~mL} / \mathrm{min}: \\
\text { Reducir dosis al } 25 \% \\
\mathrm{CICr}<30 \mathrm{~mL} / \mathrm{min} \text { : contraindicada; usar HNF }\end{array}$ \\
\hline Dalteparina & 5,000 UI SC cada $24 \mathrm{~h}$ & $\begin{array}{l}100-139 \mathrm{~kg}: 7,500 \text { UI SC cada } 24 \mathrm{~h} \\
140-180 \mathrm{~kg}: 5,000 \text { UI SC cada } 12 \mathrm{~h}\end{array}$ & $\begin{array}{l}\mathrm{CICr} \leq 30 \mathrm{~mL} / \mathrm{min}: \\
5,000 \text { UI SC cada } 24 \mathrm{~h}\end{array}$ \\
\hline Bemiparina & $3,500 \mathrm{UI} \mathrm{SC}$ cada $24 \mathrm{~h}$ & $<60$ kg: 2,500 UI SC cada $24 \mathrm{~h}$ & $\begin{array}{l}\mathrm{CICr} \leq 30 \mathrm{~mL} / \mathrm{min}: \\
\text { Reducir dosis al } 25 \%\end{array}$ \\
\hline Tinzaparina & 50 UI/kg/ día & & $\mathrm{CICr}<20 \mathrm{~mL} / \mathrm{min}:$ contraindicada \\
\hline Fondaparinux & $2.5 \mathrm{mg} \mathrm{SC}$ cada $24 \mathrm{~h}$ & & $\begin{array}{l}\text { CICr } 20-29 \mathrm{~mL} / \mathrm{min}: \\
2.5 \mathrm{mg} \text { cada } 48 \mathrm{~h} \\
\mathrm{CICr}<20 \mathrm{~mL} / \mathrm{min} \text { : contraindicado; usar HNF }\end{array}$ \\
\hline HNF & 5,000 UI SC cada $12 \mathrm{~h}$ & $\begin{array}{l}\text { IMC }>30 \mathrm{~kg} / \mathrm{m}^{2}: \\
5,000 \text { UI SC cada } 8 \mathrm{~h}\end{array}$ & Mantener dosis \\
\hline
\end{tabular}

*IMC: índice de masa corporal; SC: subcutáneo.

Tabla 5. Factores de riesgo trombótico adicionales

\begin{tabular}{|c|c|c|c|}
\hline $\begin{array}{l}\text { Por } \\
\text { laboratorio }\end{array}$ & $\begin{array}{l}\text { PCR }>150 \mathrm{mg} / \mathrm{L} \\
\text { Dímero D: } 3 \text { veces el valor normal } \\
\text { IL-6 }>40 \mathrm{pg} / \mathrm{mL} \text {, ferritina }>1,000 \mathrm{ng} / \mathrm{mL} \text {, } \\
\text { linfopenia }<800 / \mathrm{mL}\end{array}$ & $\begin{array}{l}\text { Dímero D: } 6 \text { veces el valor normal a pesar de } \\
\text { enfermedad subyacente controlada }\end{array}$ & Calificación de CID $\geq 5$ o CIS $\geq 4$ \\
\hline Clínicos & Trombofilia conocida & $\begin{array}{l}\text { Antecedente de trombosis venosa profunda, } \\
\text { embolia pulmonar o arteriopatía isquémica }\end{array}$ & $\begin{array}{l}\text { Cáncer activo con bajo riesgo de } \\
\text { hemorragia }\end{array}$ \\
\hline
\end{tabular}

No existe una escala de riesgo trombótico o hemorrágico específica para COVID-19; se utilizan marcadores de inflamación que se han informado en relación con la infección por SARS-CoV-2, además de factores de riesgo en escalas como Padua o IMPROVE $28,29$.

adaptadas a la situación local de Latinoamérica. En los puntos controvertidos o sin acuerdo se consignan las diferentes opiniones. El documento se revisó otra vez el 8 de julio.

\section{Recomendaciones: control del riesgo de trombosis relacionada con la COVID-19}

1. Todos los pacientes hospitalizados requieren vigilancia de la coagulación al menos con hemograma, tiempo de protrombina (TP), tiempo de tromboplastina parcial activado (TTPa), fibrinógeno y dímero $\mathrm{D}$. En los pacientes en estado crítico, este seguimiento debe realizarse cada 24 a 48 h, según sean la disponibilidad de las pruebas y la capacidad del laboratorio ${ }^{19}$. Las tablas 1 y 2 muestran los criterios para el diagnóstico de coagulación intravascular diseminada $(C I D)^{20}$ y de coagulopatía inducida por sepsis $(\mathrm{CIS})^{21}$ de la International Society on Thrombosis and Haemostasis (ISTH).

2. Todos los pacientes hospitalizados con COVID-19 (excepto los que tienen hemorragia o alto riesgo de hemorragia según las guías NICE 2019 (tabla 3)22,23 requieren tromboprofilaxia. La profilaxia debe iniciarse en el momento de la hospitalización, aunque no se disponga aún del resultado de la prueba confirmatoria de SARS-CoV-2.

3. No existe evidencia en relación con la administración de ácido acetilsalicílico vinculada con la tromboprofilaxia en COVID-19. En pacientes que ya lo consumen de manera crónica para otras indicaciones se sugiere continuar con él con el seguimiento habitual. 
Tabla 6. Dosis profilácticas intermedias utilizadas en estudios clínicos de COVID-19

\begin{tabular}{|l|l|l|}
\hline Medicación & Dosis & Publicación/referencia \\
\hline Enoxaparina & $40 \mathrm{mg} \mathrm{SC}$ cada $12 \mathrm{~h}$ & SISET $^{30}$ \\
\hline Enoxaparina & $1 \mathrm{mg} / \mathrm{kg} \mathrm{SC}$ cada $24 \mathrm{~h} 0.5 \mathrm{mg} / \mathrm{kg} \mathrm{SC}$ cada $12 \mathrm{~h}$ & SETH $^{25}$ \\
\hline HBPM (no especificada) & Dosis terapéutica para profilaxia antitrombótica & Llitjos, et al. $^{18}$ \\
\hline Nadroparina & $5,700 \mathrm{lU}$ cada $12 \mathrm{~h}$ (doble de la dosis profiláctica regular) & Klok, et al. $^{12,14}$ \\
\hline
\end{tabular}

Considerar ajuste según función renal y contraindicaciones para dosis elevadas como plaquetas $<50,000 / u L$ y hemorragia.

Tabla 7. Dosis terapéuticas de HNF, HBPM y fondaparinux con ajustes para peso y función renal

\begin{tabular}{|c|c|c|c|}
\hline & Dosis terapéutica & Obesidad & Insuficiencia renal \\
\hline Enoxaparina & $1 \mathrm{mg} / \mathrm{kg} \mathrm{SC}$ cada $12 \mathrm{~h}$ & $\begin{array}{l}1 \mathrm{mg} / \mathrm{kg} \mathrm{SC} \text { cada } 12 \mathrm{~h} \\
\text { Observar función renal }\end{array}$ & $\begin{array}{l}\mathrm{CICr} 15-29 \mathrm{~mL} / \mathrm{min}: 1 \mathrm{mg} / \mathrm{kg} \text { cada } 24 \mathrm{~h} \text { o HNF } \\
\mathrm{CICr}<15 \mathrm{~mL} / \mathrm{min}: \text { evitar (usar HNF) }\end{array}$ \\
\hline Nadroparina & \multicolumn{2}{|l|}{$\begin{array}{l}<50 \mathrm{~kg}: 3,800 \text { IU SC cada } 12 \mathrm{~h} \\
50-59 \mathrm{~kg}: 4,750 \text { IU SC cada } 12 \mathrm{~h} \\
60-69 \mathrm{~kg}: 5,700 \text { IU SC cada } 12 \mathrm{~h} \\
70-79 \mathrm{~kg}: 6,650 \text { IU SC cada } 12 \mathrm{~h} \\
80-89 \mathrm{~kg}: 7,600 \text { IU SC cada } 12 \mathrm{~h} \\
\geq 90 \mathrm{~kg}: 8,550 \text { IU SC cada } 12 \mathrm{~h}\end{array}$} & $\begin{array}{l}\mathrm{CrCl} 30-50 \mathrm{~mL} / \mathrm{min}: \\
\text { Administrar } 25 \% \text { de la dosis } \\
\mathrm{CrCl}<30 \mathrm{~mL} / \mathrm{min}: \text { contraindicado }\end{array}$ \\
\hline Dalteparina & \multicolumn{2}{|c|}{$\begin{array}{l}200 \mathrm{IU} / \mathrm{kg} \text { diarias por } 30 \text { días, seguidas de } 150 \mathrm{IU} / \mathrm{kg} \text { SC el resto del tiempo } \\
\text { de tratamiento } \\
\text { (dosis máxima: } 18.000 \mathrm{IU} \text { día) } \\
\text { Reducir dosis } 17-33 \% \text { con plaquetas }<100,000 / \mathrm{uL}\end{array}$} & $\begin{array}{l}\mathrm{CrCl} \leq 30 \mathrm{~mL} / \mathrm{min}: \\
\text { Ajustar dosis con actividad anti-FXa: } \\
\text { mantener en } 0.5-1.5 \mathrm{UI} / \mathrm{mL}\end{array}$ \\
\hline Bemiparina & \multicolumn{2}{|l|}{$115 \mathrm{IU} / \mathrm{kg}$ SC diarias } & $\begin{array}{l}\mathrm{CrCl} \leq 30 \mathrm{~mL} / \mathrm{min}: \\
\text { Contraindicado }\end{array}$ \\
\hline Tinzaparina & \multicolumn{2}{|l|}{$175 \mathrm{IU} / \mathrm{kg}$ SC diarias } & $\mathrm{CrCl}<20 \mathrm{~mL} / \mathrm{min}$ : contraindicado \\
\hline Fondaparinux & $\begin{array}{l}<50 \mathrm{~kg}: 5 \mathrm{mg} \mathrm{SC} \text { cada } 24 \mathrm{~h} \\
50-100 \mathrm{~kg}: 7.5 \mathrm{mg} \mathrm{SC} \text { cada } 24 \mathrm{~h}\end{array}$ & $\begin{array}{l}>100 \mathrm{~kg}: 10 \mathrm{mg} \mathrm{SC} \text { cada } 24 \mathrm{~h} \\
\text { Observar función renal }\end{array}$ & $\mathrm{CICr}<30 \mathrm{~mL} / \mathrm{min}$ : contraindicado; usar HNF \\
\hline HNF & \multicolumn{3}{|c|}{ Dosis de carga de 80 unidades $/ \mathrm{kg}$; mantenimiento de 18 unidades $/ \mathrm{kg} / \mathrm{h}$ en infusión continua. } \\
\hline
\end{tabular}

No hay datos sobre la administración de anticoagulantes orales directos (ACOD) en pacientes hospitalizados con COVID-19. Está indicado el cambio a HBPM o HNF si lo tomaban antes de la hospitalización.

En pacientes estables puede mantenerse el tratamiento con ACOD tras considerar la variabilidad de la función renal y la interacción con otros fármacos. Se recomienda no utilizarlos si el paciente recibe antivirales o bloqueadores de IL6/IL6R por el marcado incremento de los valores plasmáticos de los $\mathrm{ACOD}^{24}$.

\section{Dosis de heparinas}

La dosis adecuada para la prevención de la trombosis se encuentra bajo revisión. Existen diferentes opiniones acerca de qué situaciones clínicas se benefician de incrementar la heparina de dosis profilácticas a dosis intermedias o terapéuticas. El riesgo de hemorragia en COVID-19 parece ser bajo, si bien no se han publicado estudios específicos sobre esta complicación. Sin embargo, se han notificado casos de hemorragia mayor, por lo que el tratamiento anticoagulante debe individualizarse, con revaloración diaria de las dosis 5,17,25-27.

4. Pacientes internados en sala general (enfermería) y sin factores de riesgo adicionales para trombosis o riesgo elevado de hemorragia. Los pacientes con COVID-19 hospitalizados, que no se encuentran en terapia intensiva, tienen un riesgo elevado de trombosis hasta de 6 a $9 \%$, una incidencia mayor que los pacientes médicos hospitalizados por otras enfermedades $(1-1.5 \%)^{17}$. Todos los individuos hospitalizados con COVID-19, sin contraindicación de anticoagulación, deben recibir una dosis profiláctica regular de heparina de bajo peso molecular (HBPM) ajustada al 
peso o IMC y a la función renal. Si no se dispone de HBPM, se puede utilizar la heparina no fraccionada (HNF) o fondaparinux (tabla 4).

5. Pacientes internados en sala general (enfermería) con factores de riesgo adicionales (tabla 5): no existe evidencia sobre la dosis anticoagulante ideal para estos pacientes. Algunos expertos mantienen dosis profiláctica regular de HBPM ajustada al peso 0 IMC y a la función renal. Otros han recomendado incrementar a dosis intermedias de heparina. No existen criterios validados para la definición de alto riesgo trombótico en COVID-19, aunque sí otras escalas para calcular el riesgo de trombosis en enfermos hospitalizados ${ }^{28,29}$. En la tabla 6 se detallan las dosis consideradas por estudios clínicos y recomendadas por diferentes expertos y sociedades científicas ${ }^{14,18,25,30}$.

6. Pacientes con ventilación mecánica sin sospecha ni diagnóstico de trombosis. No se ha alcanzado consenso en cuanto a la dosis de HBPM, HNF o fondaparinux en pacientes críticos con ventilación mecánica sin confirmación ni sospecha de TEV agu$\mathrm{da}$, pese a que algunos grupos se hallan a favor de considerar las dosis terapéuticas en las siguientes situaciones: ventilación mecánica invasiva, hipoxemia resistente, inestabilidad hemodinámica vinculable a la enfermedad subyacente o una calificación de coagulopatía inducida por sepsis (CIS) $\geq 4^{13,25}$.

7. Pacientes con sospecha o diagnóstico de trombosis. La dosis terapéutica de HBPM, HNF o fondaparinux se indica en casos de sospecha o confirmación de TEV aguda o en pacientes ya anticoagulados.

8. Pacientes que con alta hospitalaria: es razonable mantener la profilaxis antitrombótica después del egreso hospitalario en el paciente con alto riesgo de trombosis o inmovilización. El riesgo hemorrágico debe ponderarse y reevaluarse de forma periódica.

Las dosis terapéuticas de heparinas se describen en la tabla 7. Se recomienda disminuir a dosis profilácticas (tabla 4) con plaquetas $<50,000 / \mathrm{L}$ y suspender con plaquetas $<25,000 / L$ o si existe hemorragia.

\section{Estudios de imagen y laboratorio}

9. La prolongación de TTPa y TP en pacientes sin hemorragia no contraindica la tromboprofilaxis.

10. En pacientes con HNF se recomienda seguimiento con unidades inhibitorias de factor Xa (concentración de 0.3-0.7 Ul/ml), de preferencia sobre el uso de TTPa, ya que este último se puede encontrar acortado por valores altos de factor VIII y fibrinógeno o prolongado si el paciente desarrolla CID.

11. Debe intentarse establecer el diagnóstico objetivo de episodios tromboembólicos por estudios de imágenes validados (angiotomografía, angiorresonancia, ecografía Doppler de las extremidades inferiores), excepto en condiciones que impidan su realización. La valoración se efectúa de modo periódico, de acuerdo con la valoración de cada enfermo y la sospecha clínica.

12. En los casos en que no pueden realizarse las pruebas diagnósticas por imágenes, el diagnóstico de episodios tromboembólicos puede sospecharse en función del historial clínico, la exploración física y las pruebas sustitutivas que sugieren sobrecarga ventricular derecha o hipertensión pulmonar, como ecocardiograma o estudios de laboratorio: incremento de troponina, pro-BNP y dímero $D$ sin incremento concomitante de PCR o ferritina. La dilatación e hipocinesia regional del ventrículo derecho (signo de McConnell) sugieren embolia pulmonar ${ }^{31}$. Se debe considerar que, en la sobrecarga repentina aguda de la presión del ventrículo derecho (VD), las presiones pulmonares calculadas podrían ser normales por falta de tiempo para compensación. Se deben descartar otras causas de hipertensión pulmonar y edema pulmonar.

13 Considerar la posibilidad de consultar con los equipos de respuesta rápida en tromboembolia pulmonar (ERRTEP) si están disponibles en el hospital en los pacientes con TEV grave compleja (p. ej., tromboembolia pulmonar masiva/submasiva, inestables o trombosis venosa profunda extensa) ${ }^{32}$.

\section{Financiamiento}

Esta investigación no ha recibido ninguna beca específica de agencias de los sectores público o comercial ni tiene fines de lucro.

\section{Conflicto de intereses}

$$
\text { Ninguno. }
$$

\section{Responsabilidades éticas}

Protección de personas y animales. Los autores declaran que para esta investigación no se han realizado experimentos en seres humanos ni en animales. 
Confidencialidad de los datos. Los autores declaran que han seguido los protocolos de su centro de trabajo sobre la publicación de datos de pacientes.

Derecho a la privacidad y consentimiento informado. Los autores declaran que en este artículo no aparecen datos de pacientes.

\section{Bibliografía}

1. Report of the WHO-China Joint Mission on Coronavirus Disease 2019 (COVID-19). https://who.int/docs/default-source/coronaviruse/who-chinajoint-mission-on-covid-19-final-report.pdf: World Health Organization; 2020.

2. Zhou F, Yu T, Du R, Fan G, Liu Y, Liu Z, et al. Clinical course and risk factors for mortality of adult inpatients with COVID-19 in Wuhan, China: a retrospective cohort study. Lancet. 2020;395(10229):1054-1062.

3. Gao Y, Li T, Han M, Li X, Wu D, Xu Y, et al. Diagnostic utility of clinical laboratory data determinations for patients with the severe COVID-19. Med Virol. 2020;10.1002/jmv.25770. doi: 10.1002/jmv.25770. Online ahead of print.

4. Huang C, Wang Y, Li X, Ren L, Zhao J, Hu Y, et al. Clinical features of patients infected with 2019 novel coronavirus in Wuhan, China. Lancet. 2020;395(10223):497-506

5. Wang T, Chen R, Liu C, Liang W, Guan W, Tang R, et al. Attention should be paid to venous thromboembolism prophylaxis in the management of COVID-19. Lancet Haematol. 2020;7(5):e362-e363.

6. Wu C, Chen X, Cai Y, Xia J, Zhou X, Xu S, et al. Risk factors associated with acute respiratory distress syndrome and death in patients with coronavirus disease 2019 pneumonia in Wuhan, China. JAMA Intern Med. 2020;e200994. doi: 10.1001/jamainternmed.2020.0994. Online ahead of print.

7. Tang N, Li D, Wang X, Sun Z. Abnormal coagulation parameters are associated with poor prognosis in patients with novel coronavirus pneumonia. J Thromb Haemost. 2020;18 (4):844-847.

8. McGonagle D, Sharif K, O'Regan A, Bridgewood C. The role of cytokines including interleukin-6 in COVID-19 induced pneumonia and macrophage activation syndrome-like disease. Autoimmun Rev. 2020;19 (6):10253.

9. Dolhnikoff M, Duarte-Neto AN, de Almeida Monteiro RA, Ferraz da Silva LF, Pierre de Oliveira E, Nascimento Saldiva PH, et al. Pathological ev dence of pulmonary thrombotic phenomena in severe COVID-19. J Thromb Haemost. 2020, Apr 15. doi: 10.1111/jth.14844. Online ahead of print.

10. Magro C, Mulvey JJ, Berlin D, Nuovo G, Salvatore S, Harp J, et al Complement associated microvascular injury and thrombosis in the pathogenesis of severe COVID-19 infection: a report of five cases. Transl Res. 2020, Apr 15:S1931-5244(20)30070-0.

11. Thachil J, Cushman M, Srivastava A. A proposal for staging COVID-19 coagulopathy. Res Pract Thromb Haemost. 2020;6;4(5):731-6. doi: 10.1002/rth2.12372

12. Klok FA, Kruip M, van der Meer NJM, Arbous MS, Gommers D, Kant KM et al. Confirmation of the high cumulative incidence of thrombotic complications in critically ill ICU patients with COVID-19: an updated analysis. Thromb Res. 2020, Apr 30;S0049-3848(20)30157-2. doi: 10.1016/j. thromres.2020.04.041.

13. Middeldorp S, Coppens $M$, van Haaps TF, Foppen M, Vlaar AP, Muller MCA, et al. Incidence of venous thromboembolism in hospitalized patients with COVID-19. J Thromb Haemost. 2020, May 5. doi: 10.1111/ ith.14888. Online ahead of print.

14. Klok FA, Kruip M, van der Meer NJM, Arbous MS, Gommers D, Kant KM et al. Incidence of thrombotic complications in critically ill ICU patients with COVID-19. Thromb Res. 2020, Apr 10: S0049-3848(20)30120-1. doi: 10.1016/j.thromres.2020.04.013.

15. Cui S, Chen S, Li X, Liu S, Wang F. Prevalence of venous thromboembolism in patients with severe novel coronavirus pneumonia. J Thromb Haemost. 2020, Apr 9. doi: 10.1111/jth.14830. Online ahead of print.
16. Helms J, Tacquard C Severac F, Leonard-Lorant I, Ohana M Delabranche $X$, et al. High risk of thrombosis in patients with severe SARSCoV-2 infection: a multicenter prospective cohort study. Intensive Care Med. 2020, May 4;1-10. doi: 10.1007/s00134-020-06062-x. Online ahead of print.

17. Lodigiani $C$, lapichino $G$, Carenzo L, Cecconi M, Ferrazzi P, Sebastian T, et al. Venous and arterial thromboembolic complications in COVID-19 patients admitted to an academic hospital in Milan, Italy. Thromb Res. 2020;191:9-14.

18. Llitjos JF, Leclerc M, Chochois C, Monsallier JM, Ramakers M, Auvray M, et al. High incidence of venous thromboembolic events in anticoagulated severe COVID-19 patients. J Thromb Haemost. 2020 Apr 22. doi: 10.1111/jth.14869. Online ahead of print.

19. Thachil J, Tang N, Gando S, Falanga A, Cattaneo M, Levi M, et al. ISTH interim guidance on recognition and management of coagulopathy in COVID-19. J Thromb Haemost. 2020;18(5):1023-1026.

20. Taylor FB, Jr., Toh CH, Hoots WK, Wada H, Levi M, Scientific Subcommittee on Disseminated Intravascular Coagulation of the International Society on T, Haemostasis. Towards definition, clinical and laboratory criteria, and a scoring system for disseminated intravascular coagulation. Thromb Haemost. 2001;86(5):1327-1330.

21. Iba T, Nisio MD, Levy JH, Kitamura N, Thachil J. New criteria for sepsis-induced coagulopathy (SIC) following the revised sepsis definition: a retrospective analysis of a nationwide survey. BMJ Open. 2017;7(9):e017046.

22. Venous thromboembolism in over 16s: reducing the risk of hospital-acquired deep vein thrombosis or pulmonary embolism. https://www.nice. org.uk/guidance/ng89: National Institute for Health and Clinical Excellence 2018.

23. The ASH COVID Resources https://www.hematology.org/covid-19/covid-19-and-coagulopathy: American Society of Hematology 2020.

24. Testa S, Prandoni $P$, Paoletti $O$ y cols. Direct oral anticoagulant plasma levels' striking increase in severe COVID-19 respiratory syndrome patients treated with antiviral agents: The Cremona experience. J Thromb Haemost. 2020;00:1-4.

25. Recomendaciones de tromboprofilaxis y tratamiento antitrombótico en pacientes con COVID-19. https://www.covid-19.seth.es/wp-content uploads/2020/04/Recomendaciones-tromboprofilaxis-y-tratamiento-antitrombotico-pacientes-COVID-19_2020-04-29.pdf: Sociedad Española de Trombosis y Hemostasia; 2020.

26. Bikdeli B, Madhavan MV, Jiménez D, Chuich T, Dreyfus I, Driggin E, et al. COVID-19 and thrombotic or thromboembolic disease: implications for prevention, antithrombotic therapy, and follow-up. J Am Coll Cardiol. 2020. J Am Coll Cardiol. 2020 Apr 15: S0735-1097(20)35008-7. doi: 10.1016/j.jacc.2020.04.031.

27. Cattaneo M, Bertinato EM, Birocchi S, Brizio C, Malavolta D, Manzoni M, et al. Pulmonary embolism or pulmonary thrombosis in COVID-19? Is the recommendation to use high-dose heparin for thromboprophylaxis justified? Thromb Haemost. 2020 Apr 29. doi: 10.1055/s-0040-1712097. Online ahead of print.

28. Barbar S, Noventa F, Rossetto V, Ferrari A, Brandolin B, Perlati M, et al. A risk assessment model for the identification of hospitalized medical patients at risk for venous thromboembolism: the Padua Prediction Score. J Thromb Haemost. 2010;8(11):2450-2457

29. Spyropoulos AC, Lipardi C, Xu J, Peluso C, Spiro TE, De Sanctis Y, et al. Modified IMPROVE VTE risk score and elevated d-dimer identify a high venous thromboembolism risk in acutely ill medical population for extended thromboprophylaxis. TH Open. 2020;4(1):e59-e65.

30. Marietta M, Ageno W, Artoni A, De Candia E, Gresele P, Marchetti M, et al. COVID-19 and haemostasis: a position paper from Italian Society on Thrombosis and Haemostasis (SISET). Blood Transfus. 2020;18(3):167-169. doi: 10.2450/2020.0083-20. Epub 2020 Apr 8.

31. Konstantinides SV, Meyer G, Becattini C, Bueno H, Geersing GJ, Harjola VP, et al. ESC Scientific Document Group. 2019 ESC Guidelines for the Diagnosis and Management of Acute Pulmonary Embolism Developed in Collaboration With the European Respiratory Society (ERS). Eur Heart J. 2020:41:543-603. doi: 10.1093/eurheartj/ehz405.

32. Porres-Aguilar M, Anaya-Ayala JE, Mukherjee D, Tapson VF. Pulmonary embolism response teams in the challenging era of venous thromboembolism associated with COVID-19. J Vasc Surg Venous Lymphat Disord. 2020;8(5):898-9. doi: 10.1016/j.jvsv.2020.04.03. 